\title{
Influence of Closeness Between Languages on L2 Acquisition Through Language Transfer
}

\author{
Shuchang Liu ${ }^{1}$, Xidong $\mathrm{Fu}^{2}$, Mingshuang Duan ${ }^{3}$ \\ ${ }^{1}$ United World College Changshu China, Changshu 215500, China. *Corresponding author. Email: \\ m15757353705@163.com \\ ${ }^{2}$ Shanghai World Foreign Language Academy, Shanghai 200233, China. *Corresponding author. Email: \\ yolandafu2004@sina.cn \\ ${ }^{3}$ Tianjin Foreign Studies University, Tianjin 300011, China. *Corresponding author. Email: 799154316@qq.com
}

\begin{abstract}
This study examines how the closeness between first language and second language affects second language acquisition, in terms of writing systems. The researchers analyzed the influence by asking experiment participants to write down characters of a second language (Japanese and Korean) at first exposure, noted the accuracy rate and recorded ways of errors of the characters. Despite being hypothized to have a lower accuracy rate due to negative transfer from L1, Japanese had a higher accuracy rate as the impact of positive transfer exceeded that of negative transfer in this study; besides the accuracy rate, through analysis of patterns of writings, the study has shown the importance of transferring phenomena in SLA, as the participants were found actively making connections to their first language.
\end{abstract}

Keywords: Second Language Acquisition, Writing System, Language Transfer.

\section{INTRODUCTION}

It is a default for people that the first language (L1) of a person, more or less, has influence on their second language (L2) acquisition. Various studies have proven that L1 has influence on L2 in aspects of phonemes [1] and morphemes [2]. One step further, another research on sign language acquisition [3] focuses on studying the accuracy of English L1 speakers' first exposure to sign language. The research revealed that the iconic signs which are similar to that action or object in real life turned out to have lower accuracy than the abstract signs. Researchers attributed this phenomenon to the negative transfer from L1 to L2. We therefore were inspired by the idea of negative transfer from L1 to L2. Since the previous research is about single L2 (sign language) acquisition and was comparing the closeness of words in the two languages, we are now turning to different L2 comparison which is a different field from the previous study. We chose to compare the two L2s since there would be a clearer boundary for iconicity between, and we do not have to design a benchmark on this.

In this research, we are mainly focusing on the relation between the closeness between L1 and L2 and the negative transfer led by it. We hence put forward our basic research question: How does the closeness between the L1 and L2 influence the L2 acquisition through language transfer? In this specific research, we decided to narrow our L1 to Mandarin Chinese. Then, regarding the fact that the previous researches are mainly based on the influence on phoneme or morpheme procduction but not on written formats of languages, we narrowed our research scope to writing systems of L1 and L2. And with regard to the rarity of a logographic writing system like Chinese, we chose Japanese Kanji as our closer L2. Then considering the basic structure and the build-up of the characters of the first two languages we chose, we chose Korean as our farther L2, with also accordance to our ability of recognizing and searching for target words.

\section{LITERATURE REVIEW}

\subsection{Negative transfer between $L 1$ and $L 2$ due to the closeness of words}

We are now hypothesizing that the closer the two languages are (in certain aspects), the more language transfer would occur. According to studies done before, it has been proven that when acquiring a second language at first exposure, the closer the words are, the more negative transfer would be likely to occur. In Ortega \& 
Morgan, they made people look at different words in sign language and then asked the participants to repeat the signs [4]. Researchers found out in the experiments that the closer the signs are to the real action or objects in the real world, the easier people are going to make mistakes on the signs and do them in the way they do in real life [4]. This proved that people would overuse their real-life knowledge when acquiring abstract signing, which is how negative transfer happens. We are therefore inspired that negative transfer in the acquisition of different kinds of languages (i.e. verbal and written) may also appear to affect the L2 acquisition when the L2 appears to be similar with L1. We hence hypothesized that the closer the L1 and L2 are, the more likely negative transfer would occur during second language acquisition. And since this research is only regarding writing systems of languages, the "closeness" here would be specifically referring to the closeness between writing systems of L1 and L2.

\subsection{Different writing systems}

\subsubsection{The Chinese writing system}

The Chinese writing system is with a logographic (some call it a morphographic) script. Different from the alphabetic writing system, in the logographic writing system, each character is combined of different parts and each character can resemble different object in the real world, and is therefore difficult for learners of the language to recognize and write. In $\mathrm{Li}$, the author defined the system as a morphographic writing system, in which he described "each character almost always corresponds to one single syllable in representing speech sound" [3]. The author of the book also described Chinese as "hard to learn for foreigners" since "each character is roughly the same size in a square shape" and "unlike English, word boundaries are not marked in Chinese" [3]. The author also stated that the Chinese writing system is widely borrowed in Asia. The Japanese Kanji and the Korean Hanja and the Vietnamese hán tựk are all borrowed from the traditional Chinese script. But since both the Korean Hanja and the Vietnamese hán tựk are no longer in daily use, in the end, we chose the Japanese Kanji which is still currently in use as our closer L2.

\subsubsection{The Japanese Writing System}

The Japanese writing system is a multi-script writing system, consisting of Hiragana, Katakana, Kanji and Romaji (some also include the Su-ji system). Hiragana and Katakana are two unique syllabographic writing system used in Japan, and the Romaji is the phonemic alphabet. These conventions of the Japanese writing system are employed "in essentially separate and complementary ways in representing the Japanese language in writing" [5]. But since the other writing systems have almost no similarities with the Chinese writing system, we would focus on the Kanji writing system in this experiment. As we have discussed just before in the part of Chinese writing system, the Japanese Kanji was borrowed from the traditional Chinese writing system, and some of the words are just from traditional Chinese (e.g. 雲, 鳥, 馬, 棟, etc.). It is also easy for us to find corpus in the Kanji writing system since it is often used now and accounts for $41.3 \%$ of the 56.6 million tokens according to a study on the Asahi Newspaper [5]. On account of the closeness to the Chinese writing system and the simpleness of finding corpus, it is the ideal close L2 writing system for us.

\subsubsection{The Korean Writing System}

We chose the Korean Hangul writing system as the farther L2 writing system in this experiment. Instead of the writing system similar to Chinese, the Korean Hanja, which is not often used in the modern Korean, the Hangul is much less similar to Chinese characters and Japanese Kanji. The Korean Hanja mainly use the traditional Chinese characters while the Korean Hangul writing system was different in the way that it is usually said to be invented by King Sejong to separate from the Chinese Hanzi. Then, instead of the logographic system, the Hangul transferred into an alphabetic syllabary writing system, according to Handel [6]. In this writing system, a single character is made up of several letters, usually at least one consonant and one vowel. "The letter representing the initial consonant is usually written on the top or top left" (It can also be written at the left part). "The letter representing the vowel is written below or to the right of the initial, depending on its shape." And "the letter representing the final consonant is written at the bottom" [6]. It is therefore essentially different from the Chinese characters in the way of pronouncing and the rules of making up a character. However, due to its unique style of writing, it is still similar to the Chinese characters in the way of single characters in most foreigners' eyes. We hence chose the Hangul writing system as the farther L2 writing system.

\section{METHOD}

The overall method is that participants of the experiment saw a character on screen for a certain amount of time, and they were instructed to produce the character as accurately as possible after it disappears from the screen.

\subsection{Participants}

Sixteen volunteers including high school students, college school students and teaching assistants, from 16 to 35 years old with an average age of 20.75 , participated in this study. All of them were native speakers of Chinese with little knowledge of Japanese, traditional Chinese or Korean. 


\subsection{Test characters}

Japanese Kanji characters chosen in this experiment can be divided into three types: those that are similar to but different from simplified Chinese characters (e.g. 鳥), those that contain certain simplified Chinese characters but with some characters added (e.g. 類), and those that can be split into simplified Chinese characters (e.g. 時). As for Korean characters, those with similar stroke numbers or structure as the chosen Japanese kanji were selected. In order to prevent influence between test characters, each radical only appear once for Japanese, and adjacent Hangul characters have no overlapping components.

\subsection{Procedure}

There were two phases of the experiment: a pilot study and the actual experiment, and the pilot study was used to determine the time given to the participants to remember and produce the target characters in the later real experiment.

During the first round of pilot study, at first, a fixation cross was shown on the screen for $1000 \mathrm{~ms}$, during which the participants were asked to look at the cross. Timing of the fixation cross was determined following Ortega \& Morgan, and Carroll \& Widjaja which were studies of SLA at first exposure that both used fixation cross of $1000 \mathrm{~ms}$ in their studies[5,7],. Then, a character appeared for $5000 \mathrm{~ms}$, after which the volunteer needed to write down the character they had seen within $10000 \mathrm{~ms}$. After the first round study, the accuracy was checked and the participants were asked about how they feel about the timing, and it was found that both the timing for appearance and for writing were too long that almost every word could be produced correctly, and the participants also reported the timing being too long. After the second and third round tests with adjustments of timing, it showed that $3500 \mathrm{~ms}$ for observing and $6000 \mathrm{~ms}$ for writing was appropriate. During the pilot study, it was also found that the participants had problems with the first characters as they may have not get used to the experiment, so two characters were added at the beginning as practice characters that were not counted in the results.

The actual experiment procedure was basically identical to that of the pilot study, but with the newly determined timing and the practice characters; also, in order to avoid the influence of attention loss on the results of characters in the latter half of the presenting sequence, the participants were divided into two groups, with the characters in different orders for different groups.

\section{HYPOTHESIS}

We hypothesized that there will be more mistakes made (i.e. lower accuracy) for Japanese than for Korean, inspired by the result of a study on sign language that participants had the lowest accuracy rate in signs that are close to real-life subjects [3]. We drew an analogy from that study and hypothesized that the participants will have a lower accuracy rate as they negatively transfer from their L1 Chinese when learning Japanese, while their production process would not be interfered when learning Korean.

\section{RESULTS}

\subsection{Accuracy of the Characters}

Table 1. Numbers of Mistakes for each Character and each Component

\begin{tabular}{|c|c|c|c|c|c|}
\hline $\begin{array}{l}\text { Japanese } \\
\text { Kanji }\end{array}$ & $\begin{array}{l}\text { Mistakes } \\
\text { make }\end{array}$ & $\begin{array}{l}\text { Number of Mistakes } \\
\text { Made for Each Part of } \\
\text { the Word }\end{array}$ & $\begin{array}{l}\text { Korean } \\
\text { (Hangul) }\end{array}$ & $\begin{array}{l}\text { Mistakes } \\
\text { Made }\end{array}$ & $\begin{array}{l}\text { Number of Mistakes Made } \\
\text { for Each Part of the Word }\end{array}$ \\
\hline 鳥 & 7 & Overall & 핼 & 9 & TL:1, TR: 4, B: 7 \\
\hline 時 & 0 & 0 & 쪠 & 7 & $\mathrm{~L}: 1, \mathrm{R}: 6$ \\
\hline 類 & 9 & R: 9 & 흭 & 13 & TL: 8, TR: $6, \mathrm{~B}: 13$ \\
\hline 陽 & 4 & R: 4 & 쪁 & 10 & TL: 0, TR: 10 \\
\hline 線 & 9 & L: 7, R: 1, unclear: 2 & 퀘 & 16 & L: 13, R: 14 \\
\hline 戦 & 5 & L: $3, \mathrm{R}: 3$ & 촐 & 10 & T: 4, B: 5, Conj: 1 \\
\hline 馬 & 4 & Overall & 쪰 & 7 & TL: 0, TR: 6, B: 4 \\
\hline 臥 & 5 & $\mathrm{~L}: 3, \mathrm{R}: 2$ & 해 & 10 & L: $3, \mathrm{R}: 7$ \\
\hline
\end{tabular}




\begin{tabular}{|c|c|c|c|c|c|}
\hline 業 & 5 & B: 5 & 빽 & 12 & TL: 2, TR: $6, \mathrm{~B}: 10$ \\
\hline 奪 & 3 & $\mathrm{M}: 1, \mathrm{~B}: 3$ & 뺇 & 16 & $\begin{array}{l}\text { TL: 7, TR: } 13, \text { BL: } 14, \text { BR: } \\
15\end{array}$ \\
\hline 栃 & 3 & $\mathrm{R}: 3$ & 퇘 & 9 & TL: 6, BL: 5, R: 10 \\
\hline 岡 & 3 & In: 3 & 밯 & 9 & TL: 3, TR: 1, B: 5 \\
\hline 焼 & 5 & $\mathrm{R}: 5$ & 츚 & 13 & T: 2, M: 2, B: 9 \\
\hline 雲 & 0 & 0 & 율 & 6 & $\mathrm{~T}: 1, \mathrm{~B}: 3$ \\
\hline 斉 & 1 & B: 1 & 짙 & 9 & TL: 2, TR: 7, B: 6 \\
\hline SUM & 63 & I & SUM & 156 & I \\
\hline
\end{tabular}

(L: left, R: right, T: top, B: bottom, In: inside, M: middle, Conj: conjunction error)

According to table 2, there were much less mistakes made for Japanese than for Korean: unexpectedly, the error rate of Korean characters (65\%) was near 2.5 times that of Japanese kanji $(26.25 \%)$. The Korean characters errors were 156 times (out of 240 productions of characters in total), and the Japanese kanji errors of all participants were 63 times (out of 240 productions of characters in total), which disproved our hypothesis that participants would do better in Japanese Kanji than in Korean characters.

Besides the overall accuracy, there were also some interesting observations regarding the accuracy by

components of characters. For both languages, most mistakes were made on the right or at the bottom of the character (i.e. they have a lower accuracy rate), which are likely to be the later part to be seen or produced. However, by comparison, the accuracy by component was more balanced in Japanese, as 3 characters had more mistakes on the left side or at the top of the characters and 2 characters had mistakes in overall structure or placement, while in Korean all characters had mistakes primarily at the latter half, which may imply some difference in the process of remembering the characters (which will be further discussed in the next section).

\subsection{Numbers of Ways of Mistakes}

Table 2. Numbers of Ways of Mistakes for each Character

\begin{tabular}{|l|l|l|l|}
\hline Japanese Kanji & Ways of Making Mistakes & Korean (Hangul) & $\begin{array}{l}\text { Ways of Making } \\
\text { Mistakes }\end{array}$ \\
\hline 鳥 & 2 & 핼 & 9 \\
\hline 時 & 0 & 졔 & 6 \\
\hline 類 & 2 & 흭 & 13 \\
\hline 陽 & 1 & 쪁 & 11 \\
\hline 線 & 7 & 퀘 & 12 \\
\hline 戦 & 4 & 촐 & 9 \\
\hline 馬 & 4 & 쪰 & 6 \\
\hline 卧 & 2 & 해 & 8 \\
\hline 業 & 2 & 빽 & 12 \\
\hline 奪 & 3 & 뺇 & 16 \\
\hline 栃 & 1 & 퇘 & 11 \\
\hline
\end{tabular}




\begin{tabular}{|l|l|l|l|}
\hline 岡 & 3 & 밯 & 7 \\
\hline 焼 & 4 & 츚 & 12 \\
\hline 雲 & 0 & 율 & 4 \\
\hline 斉 & 1 & 짙 & 9 \\
\hline SUM & 36 & SUM & 145 \\
\hline
\end{tabular}

The summary of ways of mistakes already shows the drastic difference between Japanese and Korean. Here, the ratio between ways of mistakes and number of mistakes is taken for both languages, considering that the total number of mistakes are different so the number of ways of mistakes couldn't be directly compared.

Table 3. Ratio between Ways and Numbers of Mistakes

\begin{tabular}{|l|l|l|l|}
\hline$I$ & Ways of Mistakes & Numbers of Mistakes & Ratio (approx.) \\
\hline Japanese & 36 & 62 & $0.581: 1$ \\
\hline Korean & 145 & 156 & $0.929: 1$ \\
\hline
\end{tabular}

It can be seen from table 4 that the ratio between ways and numbers of mistakes is much smaller for Japanese than for Korean: it suggests approximately every incorrect character was produced twice in Japanese, while almost all incorrect characters are different in Korean.

\section{CASE ANALYSIS}

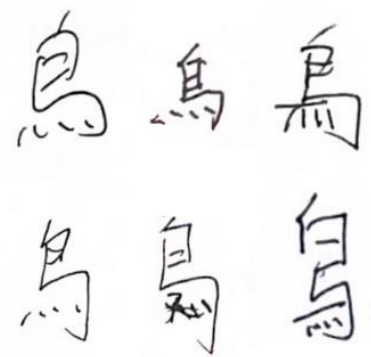

Figure 1. Example of Negative Transfer in Japanese

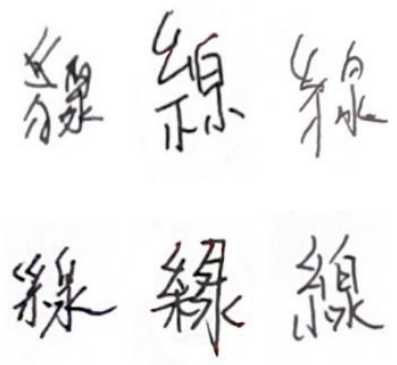

Figure 2. Example of No Transfer

Out of the characters that were commonly mistaken, some characters show obvious signs of negative transfer, while some did not show specific language transfer. For example, in image 1, basically, all mistakes of Japanese " 鳥" were made by analogy with the simplified Chinese character "鸟" as they all had issues with the extra horizontal stroke: they either ignored the stroke as it does not exist in simplified Chinese, or moved it downwards to what a horizontal stroke exists in simplified Chinese. There were also some mistakes in Japanese showing no specific transfer, like "線" in image 2, which has mistakes that are relatively scattered, probably because of its overall complexity, along with the fact that it couldn't be recognized as any simplified Chinese character. Even though the character does appear to be similar to its traditional Chinese counterpart, as we made sure the participant had little knowledge in traditional Chinese, it is still likely that they were unable to recognize this character.

Despite the difference between Chinese and Korean writing systems, negative transfer can also be found in the Korean characters the participants wrote. For example, in image 3, the bottom components of “율”, “촐”, and “핼” are very similar to Chinese “己” except for the hook at the end. However, many of the participants produced this part with a hook at the end like what they do in Chinese. Though production of this feature was not considered a mistake (if not accompanied by other errors), they are obviously signs of transferring and shows how the participants were trying to draw analogy from their L1. 


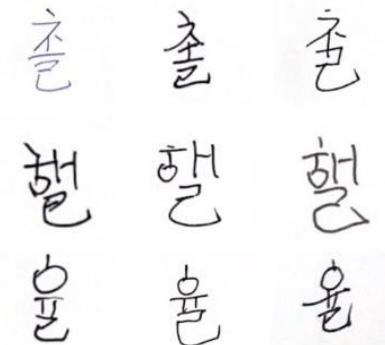

Figure 3. Example of Negative Transfer in Korean

\section{DISCUSSION}

\subsection{Discussion on the Three Main Results}

There were three main results in the previous section: first, the participants were more accurate in producing Japanese Kanji than Korean Hangul; second, they made more balanced mistakes regarding different parts of a character, when producing Japanese, they made significantly more mistakes in the later half when producing Korean; third, they had less ways of making mistakes in.

The first result has disproved the hypothesis predicting a higher accuracy rate for Korean, and the reason behind it is the crucial role of positive transfer in second language acquisition, which we did not pay enough attention to at the hypothesizing stage. From the case of transferring in Korean, it could already be seen that even when given a L2 that is fairly distanced from their L1, the participants would aim to apply their knowledge of L1 through language transfer, especially in components of the characters. Given this fact that the participants were actively trying to transfer, and that the Japanese Kanji system is highly related to that of Simplified Chinese, it is reasonable that there was a higher accuracy rate for Japanese through positive transfer.

The second result is a bit complicated and may not be fully explained, but further improvements will be provided to investigate this issue. It is possible that the characters had a lower accuracy on the later part in both languages as both the target languages and the first language of the participants are usually written from left to right, and the participants recalled the characters following this reading habit. However, there were counterexamples of this phenomenon in Japanese while all characters follow this pattern in Korean. This, together with the previous analysis that the participants aim to transfer, imply that the Japanese characters may have been remembered as a whole word, while the Korean ones were remembered as separate pieces of shapes. But since there were no solid evidence on the participants thought process, this inference may need to be further testified through incorporating apparatus such as eye tracker to better understand the participants' behaviors.

The third result provides an interesting insight after the original hypothesis being disproved by the first result. The relatively consistent mistakes in Japanese suggests that there is likely to be a common source of mistake. From the previous discussion and the cases of actual mistakes, it can be reasonably inferred that the source is the participants' L1, as they were referring to the same Chinese character when producing the Japanese one. On the other hand, the randomness of mistakes in Korean suggests that the participants were simply not able to recall the whole character due to memory issues, for example, the capacity of their working memory.

\subsection{Discussion on the Three Production Processes}

The results have shown that the participants were aiming to refer to their L1 for both $\mathrm{L} 2 \mathrm{~s}$, and the actual production process could be divided into three categories, according to the similarity between the character in L1 and L2.

When the character in L2 is directly composed of characters or radicals in L1, the production would be influenced by positive transfer. This mainly happens with Japanese Kanji due to its high similarity with Chinese writing system: for example, the only two characters that had no mistakes made on them, 時 and 雲, are both directly composed of simplified Chinese characters. Here, the participants recognized the parts as the same as their L1, and they are indeed the same in simplified Chinese, so the language transfer is positive and helps with their character production.

When the character in L2 is composed of characters in L1 but with slight differences, negative transfer is likely to happen. This mainly happens with Japanese, but could also be found in some of the Korean characters when the components are similar to Chinese characters. The cases of "鳥一乌" in image 1 and "己-己” in image 3 are typical examples of this. Here, the participants recognized the parts as the same with their L1, but they are actually different due to the small differences, so the language transfer is negative and lead to mistakes in their character production.

When the character in L2 cannot be recognized as any character in L1, no language transfer happens, and the mistakes are due to memory issues. This happens with most Korean, but could also be found in some Japanese characters that are complex or very different from their Chinese counterparts. For example, the character “線” in image 2 could hardly be recognized as its simplified Chinese counterpart “线”, which shows why its mistakes were relatively random. 


\subsection{Evaluation and Improvements}

There are several adjustment that could be made to this experiment due to some issues found in the analysis, in order to improve the experiment accuracy.

The first issue is that the participants sometimes refer backwards to their previous answers and simply copy them, as they thought there were repeated characters (during the experiment, many of the participants explicitly asked whether the characters were repeated), which leads to problems as they were not referring to their memory directly. The issue may be fixed by making a slight change to the experiment procedure: instead of letting the participants write on the same piece of paper through the entire process, it may be helpful to have separate flashcards for different characters and to only let the participants have the card for the character they are writing. In this way, they will not be able to copy from the previous characters.

The second issue is that the writing system is very different from the other components of a language (e.g. phonetics/phonology, syntax), as there is a prescriptive rule and even L1 speakers can make spelling mistakes in writing their first language. In one character in the experiment, 臥, the participants added an extra vertical stroke in the left part of the character, as shown in image 4; however, this line was not present in its simplified Chinese counterpart 卧, so this is a result of spelling mistake. The simple solution of this would be to research on the commonly mistaken characters in participants' L1 and avoid using these characters in the experiment to minimize confusion.

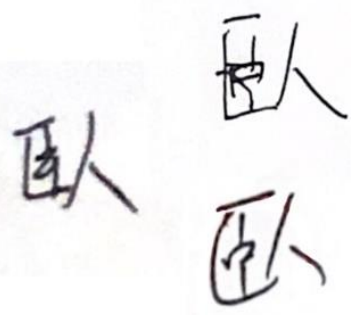

Figure 4. Example of Character with "Spelling Mistake"

\section{CONCLUSION}

In this research regarding closeness between languages and L2 acquisition at first exposure regarding language transfer, we found out that the participants do better in producing Japanese Kanji than Korean Hangul scripts, they have less ways of of making mistakes in Japanese than Korean, and in Japanese they make mistakes in a more balanced way regarding different parts of the character. Even though the original hypothesis was rejected, the result of this experiment has clearly shown how second language learners were actively trying to make connections to their first language, regardless of the closeness between L1 and L2. However, the closeness between L1 and L2 does play an important role in SLA, as language transfer, both positive and negative, is much more likely to happen with languages that are close (at least in certain aspects) to each other.

\section{REFERENCES}

[1]. Polka, L., \& Werker, J. F. (1994). Developmental changes in perception of nonnative vowel contrasts. Journal of Experimental Psychology: Human Perception and Performance, 20(2), 421-435. https://doi.org/10.1037/0096-1523.20.2.421

[2]. Vokic, G. (2010). L1 Allophones in L2 Speech Production: The Case of English Learners of Spanish. Hispania, 93(3), 430-452.

[3]. Li, Y. (2019). The chinese writing system in asia: An interdisciplinary perspective. Routledge.

[4]. Ortega, G., \& Morgan, G. (2015). Input processing at first exposure to a sign language. Second Language Research, 31(4), 443-463. https://doi.org/10.1177/0267658315576822

[5]. Pae, H. K. (2018). Writing systems, reading processes, and cross-linguistic influences. Reflections from the Chinese, Japanese and Korean languages. John Benjamins Publishing Company.

[6]. (2016, June 1). Learn to read Korean: An introduction to the hangul alphabet. http://jacow.org/ipac2016/doi/JACoW-IPAC2016THEA01.html

[7]. Carroll, S. E., \& Widjaja, E. (Nina). (2013). Learning exponents of number on first exposure to an L2. Second Language Research, 29(2), 201-229. https://doi.org/10.1177/0267658312473471 